\title{
Contribution to the discussion of current state and future perspectives of sensory analysis of balsamic vinegars
}

\author{
Efimia Hatzidimitriou, Maria Papadopoulou, Sofia Lalou, Maria Z. Tsimidou \\ Aristotle University of Thessaloniki, School of Chemistry, Laboratory of Food Chemistry \\ and Technology, Greece
}

\section{Dear Editor,}

Motivated by the discussion held in the published review of Lemmetti et al. ${ }^{1}$ addressing the current state and future perspectives of traditional balsamic vinegars' (TBVs) sensory analysis, we would like to contribute with our experience regarding sensory evaluation of certain products belonging to the balsamic family. ${ }^{2}$ Balsamic vinegars (BVs), elaborated by blending concentrated and/or boiled grape must, wine vinegar and permitted additives, are by consequence much less expensive than TBVs and are rapidly emerging worldwide.

At the time we started our research, scientific studies related to vinegar sensory evaluation were limited to wine, sherry and TBVs. Regarding the latter, sensory descriptors are individually assessed by specifically trained registered experts and a final score leading to quality ranking is calculated. As no such panels exist in Greece, we investigated the possibility to train wine experts and build up a panel for BV assessment. After a preliminary session, wine experts declared unavailable and unwilling to continue for obvious reasons. Our work continued with selection and training of novice assessors. Degree of motivation, commitment and strong liking for sour tastes were determining criteria in the recruitment of candidates that had to face the irritating pungency caused by the high content of acetic acid.

A generic descriptive analysis designed to meet our needs was chosen. ${ }^{3}$ A pre-defined list of 34 descriptors that assembled terms from literature for all types of vinegar as well as attributes proposed by the wine experts, was initially provided. Standard sensory evaluation protocols $s^{4,5}$ were proved very important for reliable results in agreement with the comments of Lemmetti et al. ${ }^{1}$ During training, assessors suggested additional terms that adequately described and differentiated the examined products. After synonyms, irrelevant or ambiguous

\footnotetext{
Correspondence: Efimia Hatzidimitriou, Aristotle University of Thessaloniki, School of Chemistry, Laboratory of Food Chemistry and Technology, 54124, Thessaloniki, Greece.

Tel.: +302310997823 - Fax: +302310997779 .

E-mail: effiehd@chem.auth.gr

Key words: Letter to the Editor; balsamic vinegars; sensory analysis.

Received for publication: 27 January 2015.

Revision received: 3 March 2015

Accepted for publication: 15 April 2015.

This work is licensed under a Creative Commons Attribution 3.0 License (by-nc 3.0).

(C) Copyright E. Hatzidimitriou et al., 2015

Licensee PAGEPress, Italy

Acetic Acid Bacteria 2015; 4:5070

doi:10.4081/aab.2015.5070
}

terms were discarded through a consensus procedure and the assistance of reference standards, a revised list of 40 terms came up. Further trials and multistep statistical analysis were necessary to reach a final operational list of 15 descriptors. ${ }^{6}$ Among them, six terms (Caramel, Raisin, Wood, Sweetness, Bitterness and Acidity) were common with those in the lexicon for TBVs that appeared in literature in the meantime. ${ }^{7}$ Pungency, Aftertaste, Tannic, Sundried tomato, Red fruits, Ethyl Acetate, Quince, Coffee and Tapenade, in descending order of importance, were additionally retained. Regarding the latter, some interesting observations and remarks can be made. The first concerns the term Sundried tomato. Sundried tomato was used in the work of Hongsoongnern and Chambers ${ }^{8}$ as reference standard for the aroma and flavor attributes browned and fermented defined as Dark impression often associated with toasted and caramelized and Aromatics associated with ripe/overripe fruit; can be somewhat sweet, sour, browned and fruity, respectively. The authors developed a lexicon to describe the sensory characteristics of various tomato products. Interestingly, the attribute Sundried tomato included in the lexicon for BVs proposed by our panel undoubtedly assembles all of the above characteristics. Moreover, the same term was also used, accompanied by savoury, to describe wines from the Pinot Noir red grape variety. ${ }^{9}$ The terms Red fruits, Quince, Coffee and Tapenade were used to evoke positive simple or more complex aromas reminiscent of fruits like cherries or strawberries, quince jelly, roasted coffee and mature olives. The panelists associated these aromas with the grape variety used for wine making as well as grape must concentration through the boiling process. Aftertaste was defined like in wine, as the taste lingering in the mouth after the vinegar is tasted. We strongly believe this term can help as a quality feature to differentiate various categories of BVs. On the other hand, Pungency, Tannic and Ethyl Acetate were proposed as negative contributors referring to the aggressiveness provoked by acetic acid, rough tannins and ethyl acetate, respectively.

Application of the lexicon to a set of randomly chosen Greek and Italian commercial products permitted the sensorial differentiation between BVs and BVs of Modena. ${ }^{10}$ Higher scores for Acidity, Pungency and Tannic and lower ones for Sweetness characterized the former while Sweetness, Raisin, Red Fruit and Caramel prevailed in the latter. Chemical data related to gustatory attributes supported the perceived sensorial differences with regard to sugars while the trend was less obvious for acidity. Nevertheless, sourness is a complex sensation and detailed acid composition and equilibria need to be considered before relating sensory to analytical data. In the same direction, we support the use of bipolar scale proposed by Lemmetti et al. ${ }^{1}$ If such scale is adopted, we would like to stress the need for a scoring that differentiates negative from positive contribution of the same attribute when in excess, as in Pungency for example.

In conclusion, our study pointed out the difficulties met during lexicon development for sensory evaluation of BVs. The extra positive and negative attributes found to characterize also the sensory properties of 
the new generation of BVs may be of help in the development of an expanded lexicon as also suggested by the pioneers Giudici et al. ${ }^{11}$

\section{References}

1. Lemmetti F, Solieri L, Bonciani T, et al. Sensory analysis of traditional balsamic vinegars: current state and future perspectives. Acetic Acid Bacteria 2014;3:4619-24.

2. Giudici P, Gullo M, Solieri L, Falcone PM. Technological and microbiological aspects of traditional balsamic vinegar and their influence on quality and sensorial properties. Adv Food Nutr Res 2009;58:137-82.

3. Lawless HT, Heymann H. Descriptive analysis. In: Lawless HT, Heymann H, eds. Sensory evaluation of food, principles and practices. New York: Springer; 2010. pp. 227-257.

4. Tesfaye W, Morales ML, Callejon RM, et al. Descriptive sensory analysis of wine vinegar: tasting procedure and reliability of new attributes. J Sens Stud 2010;25:216-30.

5. ISO (International Organization for Standardization). ISO 3591:1977. Sensory analysis - Apparatus - Wine-tasting glass. Geneva: International Organization for Standardization
Publications; 1977.

6. ISO (International Organization for Standardization). ISO 11035:1994. Sensory analysis-Identification and selection of descriptors for establishing a sensory profile by a multidimensional approach. Geneva: International Organization for Standardization Publications; 1994.

7. Zeppa G, Gambigliani Zoccoli M, Nasi E, et al. Descriptive sensory analysis of Aceto Balsamico Tradizionale di Modena DOP and Aceto Balsamico Tradizionale di Reggio Emilia DOP. J Sci Food Agric 2010;93:3737-42.

8. Hongsoongnern P, Chambers E IV. Lexicon for texture and flavor characteristics of fresh and processed tomatoes. J Sens Stud 2008;23:583-99.

9. Parr WV, White KG, Heatherbell DA. The nose knows: influence of colour on perception of wine aroma. J Wine Res 2003;14:79-101.

10. Lalou S, Papadopoulou M, Hatzidimitriou E, Tsimidou MZ. Contribution to a descriptive sensory analysis lexicon for the evaluation of commercial balsamic vinegars. 1st IMEKOFOODS, Promoting Objective and Measurable Food Quality \& Safety, Rome, 12nd-15th October 2014, P29, p 117.

11. Giudici P, Falcone PM, Scacco A, Lanza CM. Analisi sensoriale dell'aceto balsamico tradizionale. Ind Bevande 2009;38:27-42. 